\title{
openheart High incidence of atrial fibrillation in patients treated with ibrutinib
}

\author{
Florian Baptiste, ${ }^{1}$ Jennifer Cautela, ${ }^{1}$ Yan Ancedy, ${ }^{2}$ Noémie Resseguier, ${ }^{3}$ \\ Thérèse Aurran, ${ }^{4}$ Laure Farnault, ${ }^{5}$ Marion Escudier, ${ }^{1}$ Chloé Ammar, ${ }^{1}$ \\ Mélanie Gaubert, ${ }^{1}$ Charles Dolladille, ${ }^{6}$ Jeremie Barraud, ${ }^{1}$ Michael Peyrol, ${ }^{1}$ \\ Ariel Cohen, ${ }^{2}$ Franck Paganelli, ${ }^{1}$ Joachim Alexandre, ${ }^{6}$ Stephane Ederhy, ${ }^{2}$ \\ Franck Thuny ${ }^{1}$
}

\begin{abstract}
- Additional material is published online only. To view please visit the journal online (http://dx.doi.org/10.1136/ openhrt-2019-001049).
\end{abstract}

To cite: Baptiste F, Cautela J, Ancedy $Y$, et al. High incidence of atrial fibrillation in patients treated with ibrutinib. Open Heart 2019;6:e001049. doi:10.1136/ openhrt-2019-001049

Received 11 March 2019 Revised 2 April 2019 Accepted 14 April 2019

\section{Check for updates}

(C) Author(s) (or their employer(s)) 2019. Re-use permitted under CC BY-NC. No commercial re-use. See rights and permissions. Published by BMJ.

For numbered affiliations see end of article.

Correspondence to Dr Franck Thuny; franck.thuny@ gmail.com

\section{ABSTRACT}

Objective Atrial fibrillation (AF) is one of the most common side effects of ibrutinib, a drug that has dramatically improved the prognosis of chronic B-cell malignancies such as chronic lymphocytic leukaemia (CLL). The true incidence of ibrutinib-related AF (IRAF) is not well known and its therapeutic management poses unique challenges especially due to the inherent risk of bleeding. We aimed to determine the incidence and predictors of IRAF, and to analyse its management and outcome.

Methods A standardised monitoring was applied at two cardio-oncology clinics in consecutive patients referred before and during ibrutinib therapy. The primary endpoint was the incidence of IRAF. The excess of AF incidence with ibrutinib was studied by comparing the incidence of IRAF with the expected incidence of $A F$ in general population and in patients with CLL not exposed to ibrutinib.

Results 53 patients were included. The incidence of IRAF was $38 \%$ at 2 years and the risk was 15 -fold higher than the AF risk in both the general population and patients with CLL not exposed to ibrutinib $(p<0.0001)$. The majority of cases occurred in asymptomatic patients within the first 6 months. Left atrial volume index $\geq 40 \mathrm{~mL} / \mathrm{m}^{2}$ at treatment initiation identified patients at high risk of developing IRAF. No major bleeding events occurred in patients on ibrutinib, although the majority of patients with IRAF were treated with anticoagulants.

Conclusions This cardio-oncology study showed that the risk of IRAF was much higher than previously reported. The majority of cases occurred in asymptomatic patients justifying close monitoring.

\section{INTRODUCTION}

Ibrutinib is an oral Bruton tyrosine kinase inhibitor that has recently revolutionised the treatment and improved the patient outcome of various chronic B-cell malignancies ${ }^{1-5}$ such as chronic lymphocytic leukaemia (CLL), the most common leukaemia in adults. ${ }^{6}$ However, among the adverse events observed, atrial fibrillation $(\mathrm{AF})$ poses unique challenges because it may influence the cardiovascular management of patients. ${ }^{7}$

\section{Key questions}

What is already known about this subject?

- Atrial fibrillation (AF) is one of the most common side effects of ibrutinib, a drug that has dramatically improved the prognosis of chronic B-cell malignancies. The incidence and predictors of ibrutinib-related AF (IRAF) is not well known in the 'real life'. Moreover, the management of this adverse event is challenging especially due to the inherent risk of bleeding with ibrutinib.

What does this study add?

- This multicentre cohort study with systematic cardio-oncology follow-up reported a 2-year rate of IRAF of $38 \%$. The left atrial volume index (LAVI) was an independent predictor of this event. No major bleeding events occurred in patients on ibrutinib, although the majority of patients with IRAF were treated with anticoagulants.

How might this impact on clinical practice?

- Our results support that the incidence of IRAF may be much higher than previously reported. The majority of cases occur in asymptomatic patients in the first few months following the initiation of ibrutinib, justifying standardised and close monitoring during this period. Measurement of LAVI may help to identify the patients at high risk. Finally, these results suggest that anticoagulants could be considered in the absence of significant bleeding risk.

While the 2-year rate of ibrutinib-related AF (IRAF) has been estimated as 10\%-14\% in previous randomised and observational studies, ${ }^{1348-14}$ the cumulative incidence of IRAF may have been underestimated because of selection bias ${ }^{14}$ and the European and American health agencies recommend periodic ECG evaluation only for patients who develop arrhythmia symptoms.

The vast majority of patients with CLL who develop $\mathrm{AF}$ are candidates for anticoagulants to prevent ischaemic stroke. ${ }^{15}$ This clinical situation is very challenging due to the inherent tendency of bleeding with ibrutinib. ${ }^{16}$ Thus, 
patients may be exposed to an increased thrombo-embolic risk in cases of undiagnosed/untreated IRAF and to an increased risk of bleeding under antithrombotic therapy. Moreover, ibrutinib is metabolised by cytochrome $\mathrm{P}$ (CYP) 450 CYP3A and is an inhibitor of $\mathrm{P}$ glycoprotein leading to interaction with many drugs such as verapamil, diltiazem, amiodarone, dronedarone, digoxin and direct oral anticoagulants. ${ }^{14}$ While no general agreements exist regarding antithrombotic therapy and the heart rhythm versus rate control strategy in patients with IRAF, cardio-oncology specialists could play a crucial role in managing this therapy-limiting side effect.

We performed a multicentre prospective study of patients treated with ibrutinib followed in cardio-oncology clinics using a standardised approach to assess the incidence and predictors of IRAF, and to analyse its management and prognostic influence.

\section{METHODS \\ Patients}

This prospective cohort study was conducted in the cardio-oncology clinics of two university-affiliated adult tertiary care hospitals in France, which are cardio-oncology referral centres for those respective regions. These two clinics operate in the same way, in close collaboration with haematology/oncology departments and with the application of standardised monitoring protocols for patients undergoing cancer treatment. According to these protocols, all patients planned to receive ibrutinib are referred to dedicated cardio-oncology consultations.

From January 2015 to April 2018, all consecutive patients referred to these clinics before or during ibrutinib therapy were eligible for entry into the study. Patients on ibrutinib for more than 1 month and those who had no follow-up after the first cardio-oncology consultation were excluded. The study was approved by the institutional review board, and informed consent was obtained from all participating patients.

\section{Baseline data and follow-up}

After being referred by a haematologist, each patient received cardio-oncology consultations, including a clinical examination, ECG and transthoracic echocardiography (TTE). A standardised case report form was completed at the end of each consultation. All clinicians were required to screen for $\mathrm{AF}$ using pulse palpation at haematology consultations and ECG at cardio-oncology consultations. Once the first cardio-oncology consultation was completed, cardio-oncology follow-up had to be conducted every 3 months.

For each patient, the systemic thromboembolic and haemorrhagic risks were estimated by the $\mathrm{CHA}_{2} \mathrm{DS}_{2}-$ Vasc [Congestive Heart failure, hypertension, Age $\geq 75$, Diabetes, Stroke, Vascular disease, Age 65-74, and Sex (female)] and HAS-BLED [Hypertension, abnormal renal/liver function, stroke, bleeding history or predisposition, labile INR, elderly (>65years), drugs/alcohol] scores, respectively. ${ }^{17}$ During TTE, the following data were collected: left ventricular ejection fraction, using the Simpson biplane method; left atrial volume index (LAVI), using the surface-length formula indexed to the body surface area; valvular heart disease quantification ${ }^{18}$ and left ventricular global longitudinal strain, using speckle tracking imaging techniques. ${ }^{19}$

ECG monitoring with 24 hours Holter or 7-day advanced external loop recorder (SpiderFlash-T, Sorin Group, Livanova) monitoring was performed at the

\begin{tabular}{|c|c|}
\hline Total population & $\mathrm{N}=53$ \\
\hline \multicolumn{2}{|l|}{ Clinical features } \\
\hline Age, median (IQR), years & $70(66-76)$ \\
\hline Sex, male n (\%) & $39(74)$ \\
\hline BMI, median (IQR) & $25(21-27)$ \\
\hline AF history, ${ }^{*}(\%)$ & $6(10)$ \\
\hline Hypertension, n (\%) & $19(36)$ \\
\hline Diabetes mellitus, $n(\%)$ & $6(11)$ \\
\hline Coronary artery disease, n (\%) & $2(4)$ \\
\hline Valvular heart disease, $\mathrm{n}(\%)$ & $0(0)$ \\
\hline Heart failure history, $n(\%)$ & $2(4)$ \\
\hline Pacemaker, n (\%) & $1(2)$ \\
\hline Smoking, n (\%) & $14(26)$ \\
\hline Obstructive sleep apnoea, n (\%) & $0(0)$ \\
\hline Thyroid disease, n (\%) & $1(2)$ \\
\hline Stroke history, n (\%) & $2(4)$ \\
\hline Major bleeding history, n (\%) & $1(2)$ \\
\hline $\mathrm{CHADS}_{2}-\mathrm{VA}_{2} \mathrm{SC}$ score, median (IQR) & $2(1-3)$ \\
\hline HAS-BLED score, median (IQR) & $1(1-2)$ \\
\hline \multicolumn{2}{|l|}{ Antithrombotic therapy } \\
\hline Antiplatelet therapy, n (\%) & $7(13)$ \\
\hline Anticoagulant therapy n (\%) & $4(8)$ \\
\hline \multicolumn{2}{|l|}{ Malignancy } \\
\hline CLL, n (\%) & $38(72)$ \\
\hline Waldenström disease, n (\%) & $8(15)$ \\
\hline Mantle lymphoma, n (\%) & $4(8)$ \\
\hline Marginal zone lymphoma, n (\%) & 1 (2) \\
\hline Diffuse large B-cell lymphoma, n (\%) & $2(4)$ \\
\hline Prior oncological treatment, $\mathrm{n}(\%)$ & $51(96)$ \\
\hline \multicolumn{2}{|l|}{ Echocardiography } \\
\hline LAVI, median (IQR), mL/m² & $32(28-40)$ \\
\hline LVEF, median (IQR), \% & $61(57-66)$ \\
\hline Global longitudinal strain† (\%) & $19(-21--17)$ \\
\hline
\end{tabular}

${ }^{*}$ First diagnosed $A F, n=1$; paroxysmal $A F, n=4$; long-standing persistent $A F, n=1$.

†Values for $n=47$ patients.

AF, atrial fibrillation; BMI, body mass index; CLL, chronic lymphocytic leukaemia; LVAI, left atrial volume index; LVEF, left ventricular ejection fraction. 
A

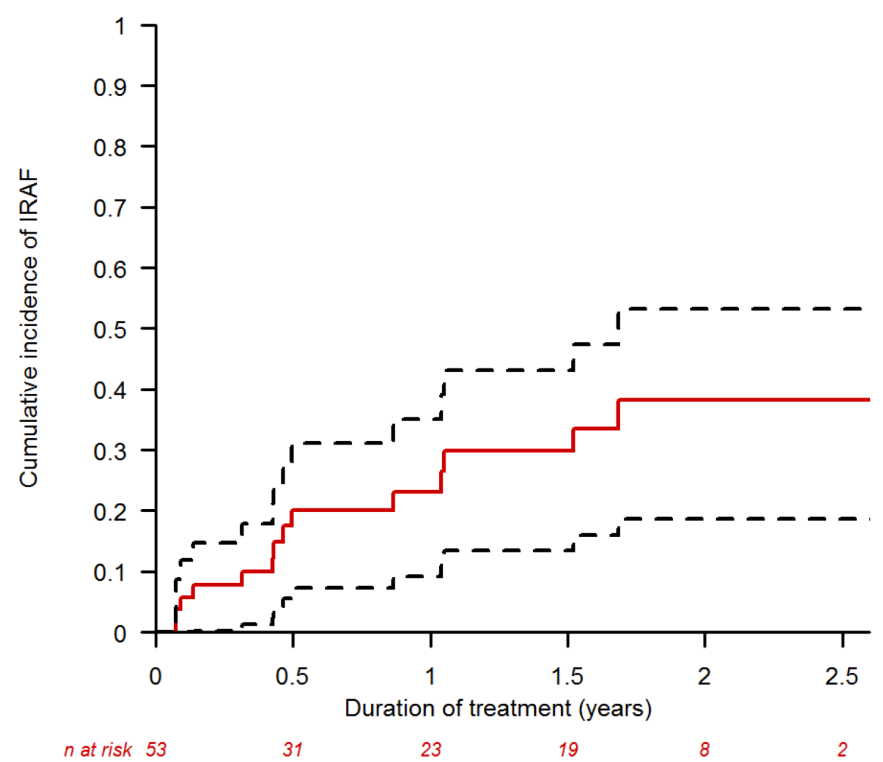

B

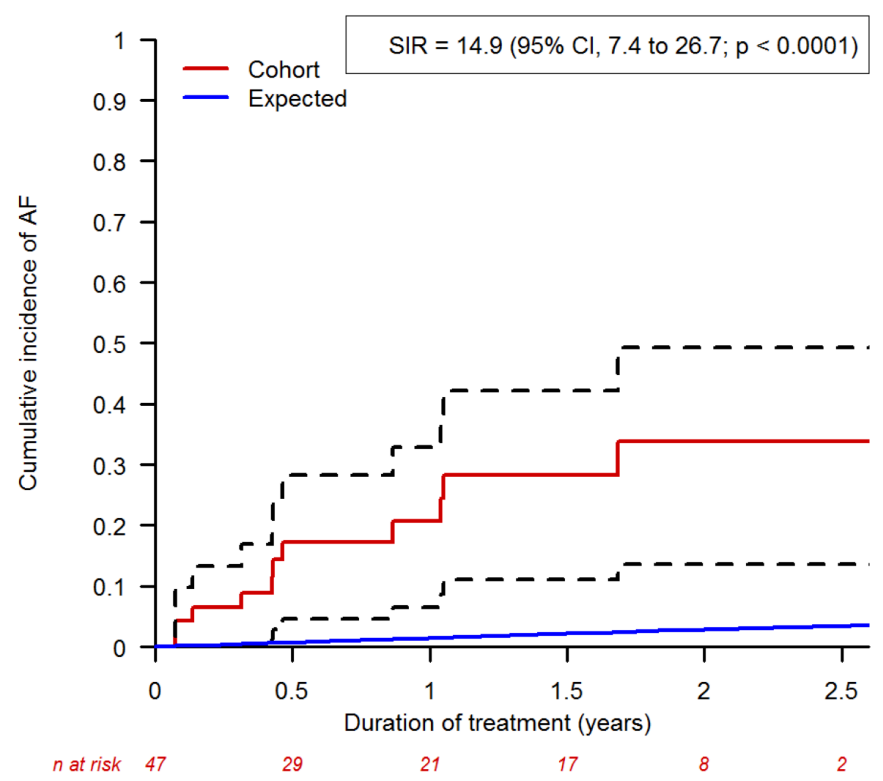

Figure 1 Cumulative incidence of IRAF in the whole cohort (A) and comparison of incidence of new episodes of AF in the cohort (Cohort) with the expected incidence in the age-comparable and sex-comparable general population (expected) (B). SIR. The dashed lines represent de $95 \% \mathrm{Cl}$ of the IRAF incidence. AF, atrial fibrillation; IRAF, ibrutinib-related atrial fibrillation; SIR, standardised incidence ratio.

discretion of the local treating clinical team, consistent with existing guidelines and local practice.

The management of haematological malignancies and cardiovascular events was left to the discretion of each centre.

\section{Study endpoints}

The primary endpoint was the incidence and timing of IRAF, which was defined as an episode of AF (or atrial flutter) on ibrutinib lasting at least $30 \mathrm{~s}$ on the ECG, 24 hours Holter ECG or long-term loop recorder pattern. An IRAF episode was defined when AF occurred after the first dose of ibrutinib in a patient with no history of AF or in a patient with a history of AF but with sinus rhythm at the time of treatment initiation. The excessive incidence of $\mathrm{AF}$ in patients on ibrutinib was studied using data from two large epidemiological studies by comparing the cumulative incidence of IRAF in patients without history of $\mathrm{AF}$ with the expected cumulative incidence of $\mathrm{AF}$ in an age-comparable and sex-comparable general population $^{20}$ and in patients with CLL not exposed to ibrutinib. ${ }^{12}$ The severity of AF episodes was defined according to the Common Terminology Criteria for Adverse Events $(\mathrm{CTCAE})^{21}$ and the modified european heart rhythm association (EHRA) score. ${ }^{17}$ Episodes were considered major for CTCAE grades of $\geq 3$. The AF pattern was defined as recommended.

The secondary endpoints were the management of antithrombotic therapy, the heart rate versus rhythm control strategy in patients with IRAF and the incidence of ibrutinib-related bleeding, the severity of which was assessed according to CTCAE classification. Bleeding was defined as major for CTCAE grades of $\geq 3$. In addition, the following cardiovascular events were analysed: the occurrence of arterial embolic events (transient ischaemic attack, stroke or peripheral ischaemia), hypertension (defined by the occurrence of de novo hypertension or the imbalance of pre-existing hypertension requiring increased medical treatment, as recommended) and death from cardiovascular causes. Finally, the prognosis of the haematological disease was assessed by analysing progression-free survival, defined as the time between the first dose of ibrutinib and the onset of haematological disease progression or death from any causes.

\section{Statistical analysis}

Quantitative data were expressed as medians (IQR), and categorical data were expressed as numbers (percentages).

Comparison of observed time-to-IRAF in the study population and expected time-to-AF in reference populations (on the one hand general population and on the other hand patients with CLL) was performed using relative survival approach, which allowed to get standardised incidence ratios (SIR) with their $95 \%$ CIs. ${ }^{22}$ Time-toIRAF analysis in the study population was conducted using Kaplan-Meier method and Cox univariate regression models to estimate crude HRs with their 95\% CIs. A multivariate Cox regression model was then proposed to assess the independent prognostic impact of a priori selected clinical data. Firth's correction was applied to take into account the small number of events. ${ }^{23}$ As all deaths occurred in patients having presented an IRAF 


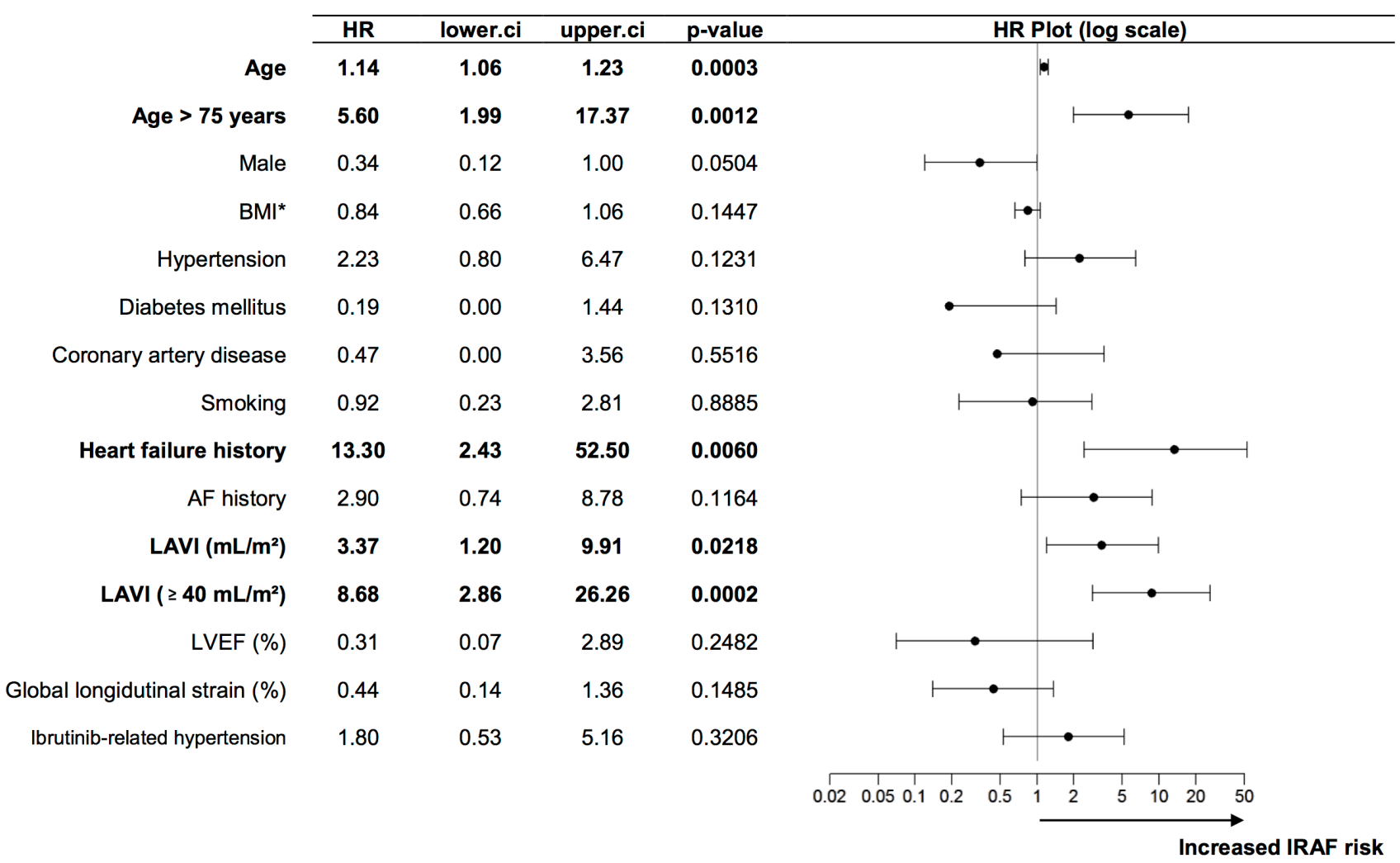

B

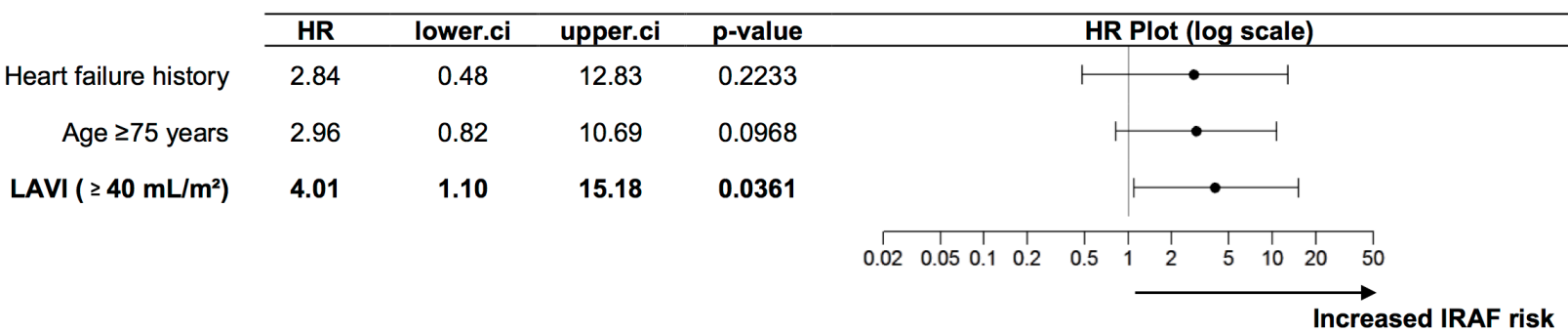

Figure 2 Predictors of IRAF by univariate (A) and multivariate (B) analyses. AF, atrial fibrillation; BMI, body mass index; IRAF, ibrutinib-related atrial fibrillation; LAVI, left atrial volume index; LVEF, left ventricular ejection fraction.

episode, competing risks approach was not applied in this setting.

Time-to-bleeding and progression-free survival analyses were also conducted using Kaplan-Meier method and Cox univariate regression models with Firth's correction. Antithrombotic therapy was considered as a time-varying covariate. The proportional hazards assumption was checked using statistical tests based on the assessment of the correlation between scaled Schoenfeld residuals and time. No multivariate models were proposed for these outcomes given the small number of events.

Statistical analysis was carried out using $\mathrm{R}$ software V.3.4.1. The following $R$ packages were used for time-to event analyses: survival, coxphf, and survexp.fr. All tests were two-sided. A p value $<0.05$ was considered to be significant.

\section{RESULTS}

\section{Baseline characteristics}

In all, 54 patients were eligible during the study period, and one was excluded due to lack of follow-up after the first cardio-oncology consultation. The baseline characteristics are summarised in table 1 . The patients had a median age of 70 years (66-76) and were predominantly men $(74 \%)$. Six patients $(10 \%)$ had a history of AF prior to the initiation of ibrutinib. Of these patients, two were receiving anticoagulation therapy (tinzaparin and rivaroxaban), one was receiving clopidogrel and three were receiving no antithrombotic treatment despite a theoretical indication.

\section{Incidence and risk of IRAF}

In all, 14 patients (26\%) experienced IRAF after a median of 13 months (4-22) and the incidence rate was 25.0 per 


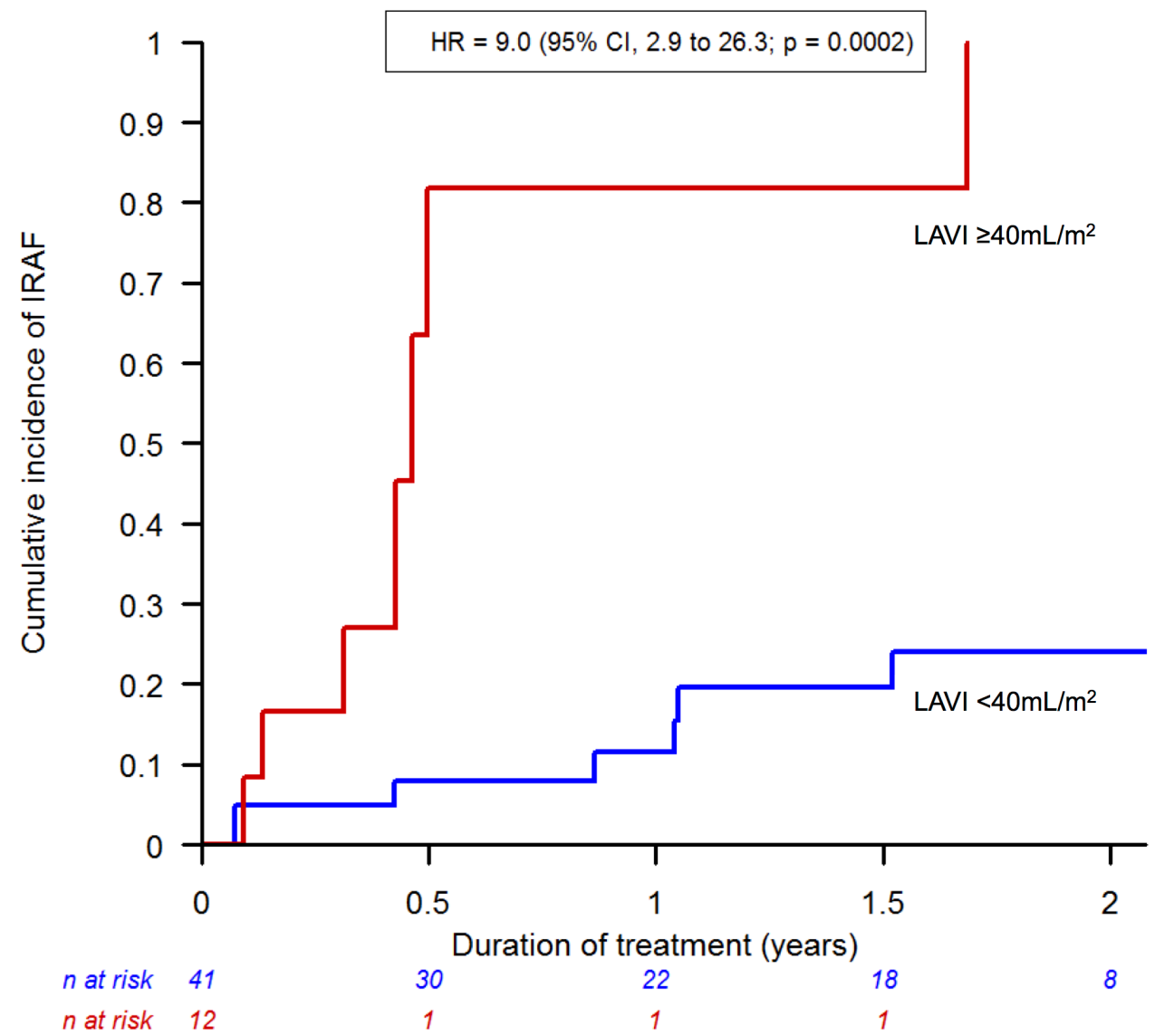

Figure 3 Cumulative incidence of IRAF according to LAVI. IRAF, ibrutinib-related atrial fibrillation; LAVI, left atrial volume index.

100 person-years. The cumulative incidence of IRAF was $21 \%$ (95\% CI, 7 to 31 ), $23 \%$ (95\% CI, 9 to 35 ) and $38 \%$ (95\% CI, 19 to 53) at 6, 12 and 24 months, respectively (figure 1A). The median time to the first event was 5.5 months (1.5-12). Episodes of IRAF were diagnosed within the first 6 months in $57 \%$ of the patients. The incidence of IRAF was significantly higher than the expected incidence of AF in the age-comparable and sexcomparable general population (SIR $=14.9 ; 95 \%$ CI, 7.4 to $26.7 ; \mathrm{p}<0.0001$ ) (figure 1B). Similarly, in the subgroup of patients receiving ibrutinib for CLL $(n=38)$, the incidence of $\mathrm{AF}$ was significantly higher than that expected in the population of patients with CLL not treated with ibrutinib (SIR $=15.0 ; 95 \%$ CI, 6.1 to $31.3 ; \mathrm{p}<0.0001$ ).

The majority of patients $(64 \%)$ were asymptomatic at the time of IRAF diagnosis. In the whole cohort, IRAF was diagnosed by ECG during cardio-oncology consultations in $79 \%$ of the cases. Holter ECG was performed in four patients, and IRAF was detected only by this method in two of these patients. Of the 17 patients who were monitored by 7-day advanced external loop recorder monitoring, all were asymptomatic, and one was diagnosed with IRAF only by this method. Four cases $(29 \%)$ of IRAF were classified as major and no cardiovascular deaths were observed in patients with IRAF.

Univariate analysis identified age, history of heart failure and LAVI as significant factors for IRAF, but LAVI of $\geq 40 \mathrm{~mL} / \mathrm{m}^{2}$ remained the only independent predictor of IRAF after multivariate analysis (figures 2 and 3 ).

\section{Management and outcome of IRAF}

The rate control strategy was selected in 10 patients $(71 \%)$ at IRAF diagnosis. Only beta-blockers were used to control heart rate. Thus, a trend towards IRAF sustainability was observed between the time of initial AF characterisation and the end of follow-up. Indeed, the incidence rates of persistent and permanent $\mathrm{AF}$ increased from $15 \%$ to $38 \%$ and from $0 \%$ to $15 \%$, respectively. Only one patient underwent electric cardioversion and then catheter ablation but IRAF recurred after 8 days.

Among the patients with IRAF, all had an indication for anticoagulation therapy as recommended. The median $\mathrm{CHA}_{2} \mathrm{DS}_{2}$-Vasc score was 2 (1-3). In all, 12 of the 14 patients with IRAF underwent anticoagulation therapy with vitamin $\mathrm{K}$ antagonists $(64 \%)$, direct oral anticoagulants $(21 \%)$ and low-molecular-weight heparin (14\%). Antiplatelet therapy was not used in any of the patients with IRAF. No antithrombotic treatment was prescribed for two patients due to a large pericardial effusion in one and significant iterative thrombocytopenia in the other. Of the 39 patients without IRAF, $18 \%$ were on antithrombotic therapy including direct oral anticoagulants $(2.6 \%)$, low-molecular-weight heparin $(2.6 \%)$ and antiplatelet drug $(13 \%)$. No major bleeding was observed during 


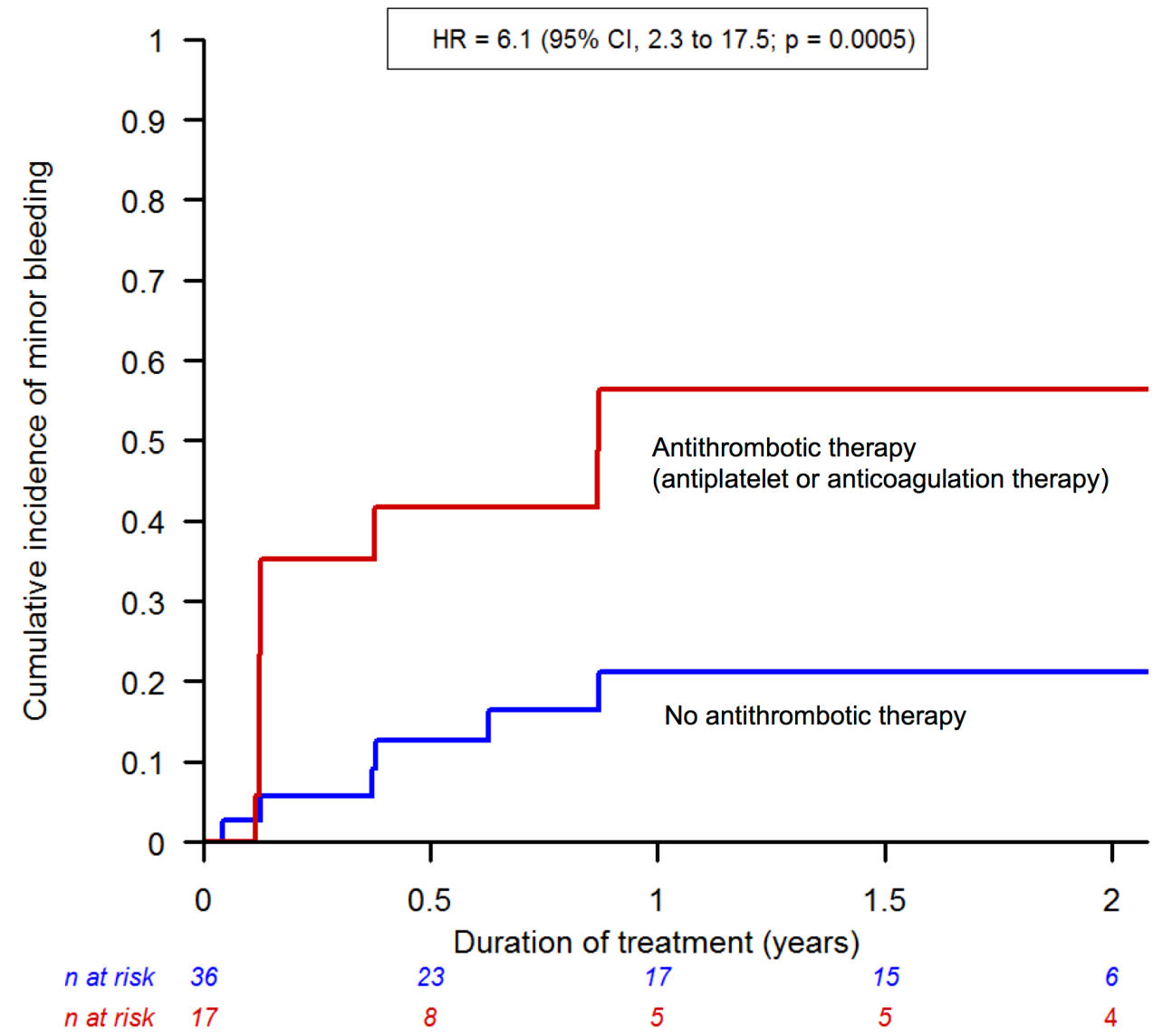

Figure 4 Cumulative incidence of minor bleeding according to antithrombotic therapy.

follow-up in any patients of the cohort. However, minor bleeding occurred in 15 patients $(28 \%)$ at a median of 1.5 months (1.5-9.1), occurring in $73 \%$ of these patients within the first 6 months. The cumulative incidence of minor bleeding was 23\% (95\% CI, 10 to 33) at 6 months and $34 \%$ (95\% CI, 18 to 47 ) at both 12 and 24 months. Of the patients who experienced bleeding, $60 \%$ were on anticoagulants and $13 \%$ were on antiplatelet therapy (online supplementary file 1). Thus, the only factor associated with the bleeding risk in the total cohort was the use of one of the two antithrombotic therapies $(\mathrm{HR}=6.1$; 95\% CI, 2.3 to $17.5 ; \mathrm{p}=0.0005$ ) (figure 4 ). Neither the presence of thrombocytopenia at the first visit $(\mathrm{HR}=1.42$; 95\% CI, 0.46 to $3.85 ; \mathrm{p}=0.52$ ) nor the HAS-BLED score (HR=1.67; 95\% CI, 0.91 to $2.91 ; \mathrm{p}=0.09)$ were predictive of bleeding events.

The occurrence of IRAF led to the temporary suspension of ibrutinib therapy in $29 \%$ for a median of 8 days (5-16), a dose reduction $14 \%$ and definitive suspension in no patients (see online supplementary file 2). The details of IRAF management are summarised in online supplementary file 3 .

No embolic events were observed in the cohort. Ten patients $(19 \%)$ experienced hypertension related to ibrutinib. In $50 \%$ of these cases, the patient had hypertension prior to ibrutinib therapy. Four episodes $(8 \%)$ of pericardial effusion were observed. No sudden deaths or episodes of ventricular arrhythmia were described. Two deaths related to haematological progression occurred. The progression-free survival rate of CLL was 92\% (95\% CI, 84 to 100) at 18 months, and IRAF was not associated with the risk of progression (figure 5).

\section{DISCUSSION}

In this multicentre prospective study, the cumulative incidence of IRAF was $38 \%$ at 2 years and the risk was 15 -fold higher than the risk of $\mathrm{AF}$ in both the general population and patients with CLL not exposed to ibrutinib. The LAVI identified patients at high risk of developing IRAF. No major bleeding events occurred in patients on ibrutinib, although the majority of patients with IRAF were treated with anticoagulants.

\section{Risk of IRAF}

Ibrutinib is a therapeutic innovation that has significantly improved the prognosis of patients with B-cell malignancies. As a result, the life expectancy of these patients is getting closer to that expected in the general population, ${ }^{1}$ giving adverse events a greater impact on long-term prognosis. AF is one of the main side effects of ibrutinib; it may lead to therapy discontinuation and is associated with major management problems, including the potential risk of bleeding with anticoagulants. Although randomised clinical trials, meta-analyses and retrospective studies have estimated that the cumulative incidence 


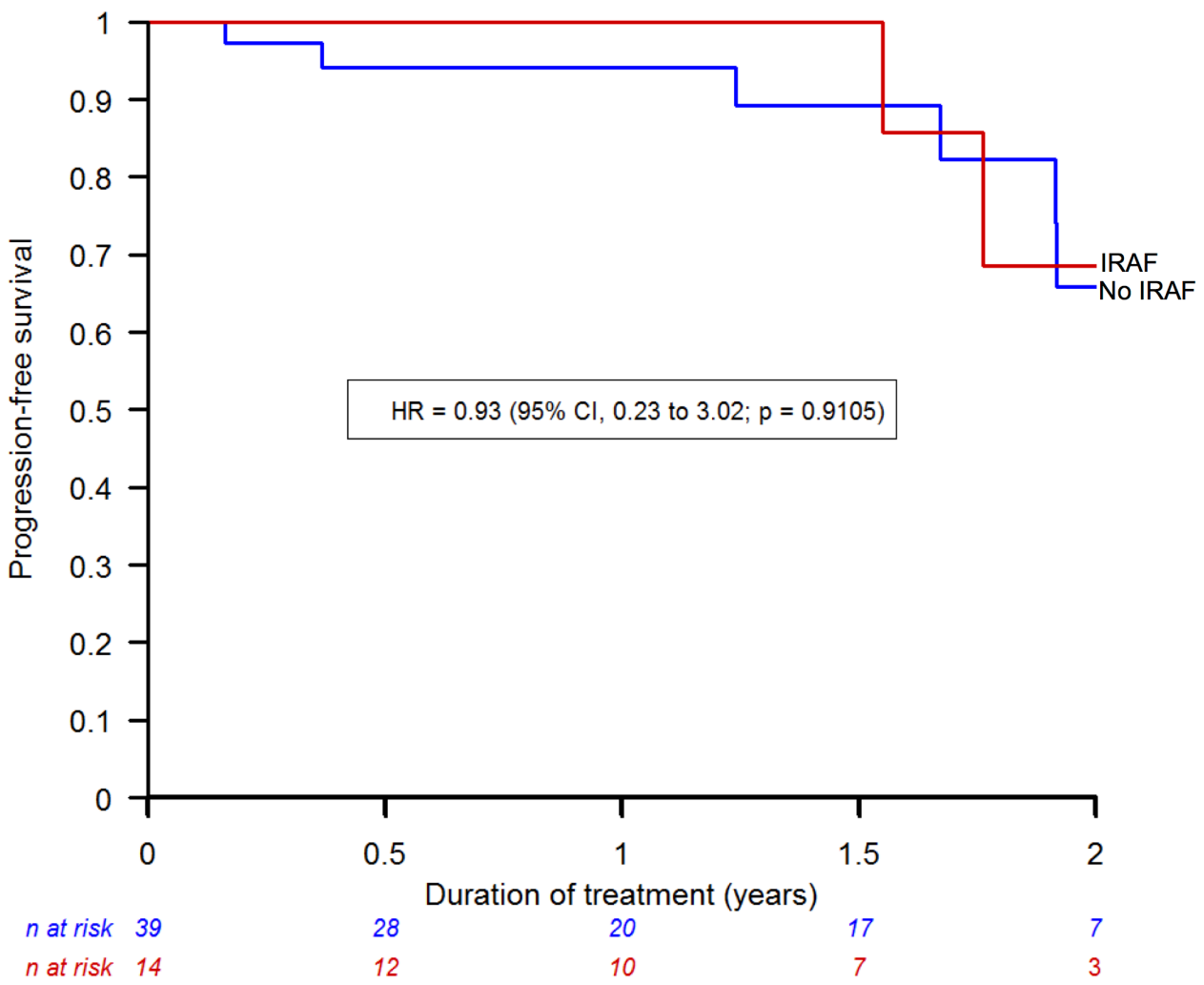

Figure 5 Progression-free survival rate according to IRAF. IRAF, ibrutinib-related atrial fibrillation.

of IRAF ranges from $10 \%$ to $14 \%$ at 2 years, ${ }^{1348-14}$ our prospective study revealed a much higher rate $(38 \%)$ at 2 years, the highest reported to date. This result can be explained by the systematic screening strategy for IRAF. Thus, IRAF was probably more diagnosed than in previous studies, especially in asymptomatic patients. No previous study used a standardised AF screening protocol; therefore, the results of this work emphasise the role of systematic cardio-oncology follow-up for patients on ibrutinib without waiting for symptoms to appear. Indeed, $64 \%$ of the patients in our study developed IRAF when they were asymptomatic. However, this study could not accurately analyse whether the use of 24 hours Holter ECG and longterm event recording was more effective than systematic ECG in screening for IRAF. Nevertheless, nearly $80 \%$ of the diagnoses were made with a simple ECG. The detection of asymptomatic IRAF by new technologies, such as smartphone applications or smart watches with AF detection algorithms, might play a role in the early diagnosis of this arrhythmia.

In clinical trials and epidemiological studies, the median age of patients treated with ibrutinib is approximately 70 years. 134891213 The risk of AF in the general population is relatively high in this age group and depends on other conditions, such as hypertension, ${ }^{25}$ which are frequently present in patients with CLL. Thus, the additional risk of AF conferred by ibrutinib treatment was not well known. Compared with published epidemiological results, the data in our study showed that the risk of developing AF with ibrutinib was 15-fold higher than that expected in the general population and in the population of patients with CLL not exposed to ibrutinib. This excess risk was higher than that hypothesised from the results of two meta-analyses, where the incidence rate was threefold to fivefold higher than in the general population. ${ }^{11} 14$

Although the pathogenesis of IRAF remains poorly understood, patients exposed to ibrutinib represent a group at very high risk of developing AF due to the association between common AF risk factors and the direct effects of treatment. In this study, after adjusting for other AF risk factors, only LAVI remained as an independent predictor for the occurrence of IRAF. Left atrial remodelling is a crucial element in the pathogenesis of AF and this observation is confirmed in patients treated with ibrutinib. Small preliminary studies previously suggested that signs of left atrial dilatation on the ECG or TTE pattern were associated with a higher risk of IRAF. ${ }^{26} 27$ However, these studies did not assess LAVI, which is the reference measure for left atrial remodelling. Thus, our result further underscores the need for a complete cardio-oncology evaluation, including the systematic measurement of LAVI, before the initiation of ibrutinib therapy and possibly proposes closer monitoring if the value is $\geq 40 \mathrm{~mL} / \mathrm{m}^{2}$. Unlike in other studies, ${ }^{13}$ a history of AF prior to the initiation of ibrutinib therapy was not independently associated with a higher risk of IRAF in our work. This result can be explained by a lack of power, but, above all, underscores the greater predictive value of left atrial remodelling than of other measures on the risk of IRAF. 


\section{Management and outcome of IRAF}

In this study, the therapeutic management of IRAF was left to the discretion of each specialist, allowing the collection of 'real-life' data. In the majority of cases, the rate control strategy with beta-blockers was favoured over the rhythm control strategy. This preference was probably related to the good clinical tolerance of most IRAF episodes but also the persistence of ibrutinib as a cause for $\mathrm{AF}$, the potential interactions with calcium channel blockers or digoxin, and the risk of recurrence after cardioversion or ablation. ${ }^{14}$ In addition, there is a risk of interaction between ibrutinib and some anti-arrhythmic therapies (eg, amiodarone) used in the rhythm control strategy, explaining the increase in the rate of persistent and permanent AF during follow-up. This management approach seems safe, considering the absence of major cardiovascular events.

Regarding antithrombotic treatment, our study showed that all patients who developed IRAF had an indication for anticoagulants and that only very few patients did not receive this treatment. More than $80 \%$ of the patients were on anticoagulants, the highest rate reported to date. Despite concerns about bleeding associated with the combination of ibrutinib plus anticoagulants, no patients experienced major bleeding events. However, our work showed that patients were exposed to an increased risk of minor bleeding, which confirms data from previous studies. ${ }^{28}$ This result suggests that anticoagulants may be considered for patients with IRAF if the thromboembolic risk is substantial and significant bleeding risk factors are absent. However, caution should be exercised during the first months of therapy, when the bleeding risk is the highest, and the anticoagulant benefit-risk ratio should be regularly reassessed, as in other patients with cancer. In our cohort, the majority of patients with IRAF were treated with vitamin $\mathrm{K}$ antagonists, probably because of concerns about interactions between ibrutinib and direct oral anticoagulants. However, recent reports suggest that oral factor Xa inhibitors may also be used for this indication. ${ }^{141529}$

\section{Limitations}

The size of our cohort prevented us from answering several questions, but our multicentre work provided 'real-life' data on the risk and management of IRAF. These data are currently scarce and the relatively small sample size is balanced by the use of a standardised cardiac-oncology monitoring protocol.

The $\mathrm{CHA}_{2} \mathrm{DS}_{2}$-Vasc score was used to estimate thromboembolic risk but was not validated in patients with malignancies. However, there is no other way to stratify embolic risk in this oncologic population. Finally, we could not compare the effect of direct oral anticoagulants versus vitamin $\mathrm{K}$ antagonists in patients with IRAF, but no major bleeding or thromboembolic events occurred with any of these treatments.

\section{CONCLUSIONS}

This prospective multicentre cohort study with systematic cardio-oncology follow-up showed that the risk of IRAF was much higher than previously reported. The majority of cases occurred in asymptomatic patients in the first few months following the initiation of ibrutinib, justifying standardised and close monitoring during this period. Measurement of LAVI may help to identify the patients at high-risk. Finally, the results suggested that the heart rate control strategy was a relatively safe therapeutic option and that anticoagulants could be considered in the absence of significant bleeding risk.

\section{Author affiliations}

${ }^{1}$ Department of Cardiology, Unit of Heart Failure and Valvular Heart Diseases, Mediterranean University Cardio-Oncology Center (MEDI-CO Center), Hôpital Nord, Aix-Marseille I University, Marseille, France

${ }^{2}$ Université Pierre et Marie Curie, Paris, France

${ }^{3}$ Biostatistic and Public Healt Department, Hopital de la Timone, Marseille, France

${ }^{4}$ Department of Haematology, Institut Paoli-Calmettes, Marseille, France

${ }^{5}$ Department of Haematology, Aix-Marseille I University, Marseille, France ${ }^{6}$ PICARO Cardio-oncology Program, Department of Pharmacology, CHRU de Caen, Caen, France

Contributors Substantial contributions to the conception and design of the work: $\mathrm{FT}, \mathrm{JC}$ and SE. Acquisition, analysis and interpretation of data for the work: $\mathrm{FB}, \mathrm{YA}$, CA, ME, MG, JB, MP, AC, FP, LB, ML, NR, TA, LF, JA and CD. Drafting the work and revising it critically for important intellectual content: FT, JC, FB, SE, LF, TA, JA and $C D$. Final approval of the version to be published: $F B, J C, Y A, N R, L F, T A, C A, M E$, $M G, J B, M P, M L, L B, A C, F P, S E, F T$, JA and CD.

Funding This study was supported by La Ligue Contre le Cancer and Assistance Publique - Hôpitaux de Marseille.

Competing interests JC received received modest consultant and lecture fees from MSD, Janssen, Merck, Novartis, Astra-Zeneca. FT received grants from Fédération Francaise de Cardiologie, Ministère français de la Santé, Fondation Coeur et Recherche, Ligue contre le Cancer, Assistance Publique - Hôpitaux de Marseille, Vifor Pharma. FT exercised an expert activity with Institut Nationale du Cancer. FT received personal fees for lectures and speakers bureaus from Abbott, Novartis, Amgen, Janssen-Cilag, Merck Sharp and Dohme, Bristol-Myers Squibb, Pfizer, Roche, Vifor Pharma, Sanofi, Astra-Zeneca. FT received non-financial support for travel and lunch paid from Abbott, Novartis, Amgen, Janssen-Cilag, Merck Sharp and Dohme, Bristol-Myers Squibb, Pfizer, Roche, Vifor Pharma, Sanofi, Astra-Zeneca, Servier, Sorin, Boston Scientific, Actelion, Bayer, Biotronik, Boehinger Ingelheim, The Medecines Compagny, Orion Pharma, Correvio, Daiichi Sankyo, St Jude Medical, Lilly, Zoll Medical, LivaNova, Medtronic, Sorin, Philips, Genzyme, Icomed, Leo Pharma, Mylan Medical, Preciphar, Resmed. AC received modest consultant and lecture fees from Astra-Zeneca, Bayer, Boehringer Ingelheim, Bristol-Myers Squibb, Daiichi Sankyo, GlaxoSmithKline, and SanofiAventis.Stephane Ederhy received modest consultant and lecture fees from Lilly, Daiichy-Sankyo, Celgene, Pfizer, Esperare, Bristol-Myers Squibb, Janssen, Philips Healthcare, Bayer, Novartis, Amgen, Ipsen.

Patient consent for publication Not required.

Provenance and peer review Not commissioned; externally peer reviewed.

Data sharing statement All data relevant to the study are included in the article or uploaded as supplementary information.

Open access This is an open access article distributed in accordance with the Creative Commons Attribution Non Commercial (CC BY-NC 4.0) license, which permits others to distribute, remix, adapt, build upon this work non-commercially, and license their derivative works on different terms, provided the original work is properly cited, appropriate credit is given, any changes made indicated, and the use is non-commercial. See: http://creativecommons.org/licenses/by-nc/4.0/. 


\section{REFERENCES}

1. Burger JA, Tedeschi A, Barr PM, et al. Ibrutinib as initial therapy for patients with chronic lymphocytic leukemia. N Engl J Med Overseas Ed 2015;373:2425-37.

2. Dreyling $M$, Jurczak $W$, Jerkeman $M$, et al. Ibrutinib versus temsirolimus in patients with relapsed or refractory mantle-cell lymphoma: an international, randomised, open-label, phase 3 study. Lancet 2016;387:770-8.

3. Treon SP, Tripsas CK, Meid K, et al. Ibrutinib in Previously Treated Waldenström's Macroglobulinemia. N Engl J Med 2015;372:1430-40.

4. Byrd JC, Brown JR, O'Brien S, et al. Ibrutinib versus ofatumumab in previously treated chronic lymphoid leukemia. $N$ Engl J Med 2014;371:213-23.

5. Byrd JC, Furman RR, Coutre SE, et al. Targeting BTK with ibrutinib in relapsed chronic lymphocytic leukemia. $N$ Engl J Med 2013;369:32-42.

6. Siegel D, Martin T, Nooka A, et al. Integrated safety profile of singleagent carfilzomib: experience from 526 patients enrolled in 4 phase II clinical studies. Haematologica 2013;98:1753-61.

7. Thorp BC, Badoux X. Atrial fibrillation as a complication of ibrutinib therapy: clinical features and challenges of management. Leuk Lymphoma 2018;59:311-20.

8. Wang ML, Rule S, Martin P, et al. Targeting Btk with ibrutinib in relapsed or refractory mantle-cell lymphoma. $N$ Engl J Med 2013;369:507-16.

9. Thompson PA, Lévy V, Tam CS, et al. Atrial fibrillation in CLL patients treated with ibrutinib. An international retrospective study. $\mathrm{Br} J$ Haematol 2016;175:462-6.

10. Yun S, Vincelette ND, Acharya U, et al. Risk of atrial fibrillation and bleeding diathesis associated with ibrutinib treatment: a systematic review and pooled analysis of four randomized controlled trials. Clin Lymphoma Myeloma Leuk 2017;17:31-7.

11. Leong DP, Caron F, Hillis $\mathrm{C}$, et al. The risk of atrial fibrillation with ibrutinib use: a systematic review and meta-analysis. Blood 2016;128:138-40.

12. Shanafelt TD, Parikh SA, Noseworthy PA, et al. Atrial fibrillation in patients with chronic lymphocytic leukemia (CLL). Leuk Lymphoma 2017;58:1630-9.

13. Wiczer TE, Levine LB, Brumbaugh J, et al. Cumulative incidence, risk factors, and management of atrial fibrillation in patients receiving ibrutinib. Blood Adv 2017;1:1739-48.

14. Ganatra S, Sharma A, Shah S, et al. Ibrutinib-Associated atrial fibrillation. JACC: Clin Electrophysiol 2018;4:1491-500.

15. Chai KL, Rowan G, Seymour JF, et al. Practical recommendations for the choice of anticoagulants in the management of patients with atrial fibrillation on ibrutinib. Leuk Lymphoma 2017;58:2811-4.
16. Rigg RA, Aslan JE, Healy LD, et al. Oral administration of Bruton's tyrosine kinase inhibitors impairs GPVI-mediated platelet function. Am J Physiol Cell Physiol 2016;310:C373-C380.

17. Kirchhof P, Benussi S, Kotecha D, et al. ESC guidelines for the management of atrial fibrillation developed in collaboration with EACTS. Eur Heart J 2016;2016:2893-962.

18. Lancellotti P, Tribouilloy C, Hagendorff A, et al. Recommendations for the echocardiographic assessment of native valvular regurgitation: an executive summary from the European association of cardiovascular imaging. Eur Heart $J$ Cardiovasc Imaging 2013;14:611-44.

19. Galderisi M, Cosyns B, Edvardsen T, et al. Standardization of adult transthoracic echocardiography reporting in agreement with recent chamber quantification, diastolic function, and heart valve disease recommendations: an expert consensus document of the European association of cardiovascular imaging. Eur Heart $J$ Cardiovasc Imaging 2017;18:1301-10.

20. Wilke T, Groth A, Mueller S, et al. Incidence and prevalence of atrial fibrillation: an analysis based on 8.3 million patients. Europace 2013;15:486-93.

21. National Cancer Institute NIoH, , US Department of Health and Human Services. Common terminology criteria for adverse events (CTCAE), version 4.0. NIH publication 09-7473, 2010.

22. Breslow N, Day N. Volume The design and analysis of cohort studies. World Health Organization, 1987.

23. Firth D. Bias reduction of maximum likelihood estimates. Biometrika 1993;80:27-38.

24. Heinze G, Schemper M. A solution to the problem of Monotone likelihood in COX regression. Biometrics 2001;57:114-9.

25. Camm AJ, Lip GY, De Caterina R, et al. Focused update of the ESC guidelines for the management of atrial fibrillation: an update of the 2010 ESC guidelines for the management of atrial fibrillation. developed with the special contribution of the European heart rhythm association. Eur Heart J 2012;2012:2719-47.

26. Mato AR, Clasen S, Pickens P, et al. Left atrial abnormality (LAA) as a predictor of ibrutinib-associated atrial fibrillation in patients with chronic lymphocytic leukemia. Cancer Biol Ther 2018;19:1-2.

27. Reda G, Fattizzo B, Cassin R, et al. Predictors of atrial fibrillation in ibrutinib-treated CLL patients: a prospective study. J Hematol Oncol $2018 ; 11$.

28. Brown JR, Moslehi J, Ewer MS, et al. Incidence of and risk factors for major haemorrhage in patients treated with ibrutinib: an integrated analysis. Br J Haematol 2018.

29. Mulligan SP, Ward CM, Whalley D, et al. Atrial fibrillation, anticoagulant stroke prophylaxis and bleeding risk with ibrutinib therapy for chronic lymphocytic leukaemia and lymphoproliferative disorders. Br J Haematol 2016;175:359-64. 